IFUM-708-FT

CAMS/02-03

hep-th/0203038

\title{
Noncommutative Gravity in two Dimensions
}

\author{
S. Cacciatori ${ }^{1,4}$, A. H. Chamseddine ${ }^{2} \pitchfork$, D. Klemm ${ }^{3,4}$, \\ L. Martucci ${ }^{3}, 4$, W. A. Sabra ${ }^{2}$, and D. Zanon ${ }^{3,4}$ \\ ${ }^{1}$ Dipartimento di Matematica dell'Università di Milano, \\ Via Saldini 50, I-20133 Milano. \\ ${ }^{2}$ Center for Advanced Mathematical Sciences (CAMS) and \\ Physics Department, American University of Beirut, Lebanon. \\ 3 Dipartimento di Fisica dell'Università di Milano, \\ Via Celoria 16, I-20133 Milano. \\ ${ }^{4}$ INFN, Sezione di Milano, \\ Via Celoria 16, I-20133 Milano.
}

\begin{abstract}
We deform two-dimensional topological gravity by making use of its gauge theory formulation. The obtained noncommutative gravity model is shown to be invariant under a class of transformations that reduce to standard diffeomorphisms once the noncommutativity parameter is set to zero. Some solutions of the deformed model, like fuzzy $\mathrm{AdS}_{2}$, are obtained. Furthermore, the transformation properties of the model under the Seiberg-Witten map are studied.
\end{abstract}

*cacciatori@mi.infn.it

†chams@aub.edu.lb

$\ddagger$ dietmar.klemm@mi.infn.it

§luca.martucci@mi.infn.it

ฯws00@aub.edu.lb

"daniela.zanon@mi.infn.it 


\section{Introduction}

Quantum field theories formulated on a noncommutative space have become recently the object of renewed interest. This is primarily due to the realization that noncommutative $U(N)$ gauge theories arise in the field theory limit of strings in a constant $B$-field background [1]. The perturbative analysis of such theories is simplified by the fact that the noncommutativity of spacetime can be traded off by a modified multiplication rule for the fields, i.e. functions on noncommutative $\mathbb{R}^{d}$ can be treated as ordinary functions on standard $\mathbb{R}^{d}$ with a deformed multiplication given by the Moyal $*$-product.

By now there exists an abundant literature on the perturbative and non perturbative studies of field theories in noncommutative flat $\mathbb{R}^{d}$. For a review and an extensive list of references cf. e. g. [2]. On the contrary, very little is known about corresponding theories in a curved noncommutative space or else about a noncommutative formulation of gravity itself. One of the main obstacles to overcome in the formulation of gravity on noncommutative spaces is related to the fact that the Moyal product does not maintain reality. One possible way to preserve nevertheless reality of the gravitational fields is to use explicitely the Seiberg-Witten map [3]. Otherwise it seems that one is forced to complexify the fields [4]. However, complex gravity may be plagued by inconsistencies already at the commutative level [7, 8].

Here we attack the problem starting from the theory of noncommutative gravity formulated in two space-time dimensions. In this case we can take advantage of our knowledge about non commutative gauge theories. There we know how to deform the gauge transformations for the fields and everything is under control at least at the kinematical level. In fact the Jackiw-Teitelboim (JT) model [9] of 2- $d$ commutative gravity can be written as a topological $S U(1,1)$ gauge theory [10,11]. Within this formulation embedding in a noncommutative space is straightforward: since in the volume form the metric does not appear it is sufficient to introduce the $*$-product appropriately and extend the gauge group to $U(1,1)$ 円. We will show how to write the action in terms of real fields and how to achieve the decoupling of the extra $U(1)$ in the commutative limit. Then we address the issue of diffeomorphisms. We find that the deformed action is invariant under a class of transformations that reproduce the standard diffeomorphisms in the commutative limit. Moreover, once the equations of motion are imposed, they are equivalent to gauge transformations, as it happens in the commutative case.

Our paper is organized as follows: in the next section we define the noncommutative gravity action in terms of a topological two-dimensional gauge theory and write the equations of motion. In section 3 it is shown that the Seiberg-Witten (SW) formula maps the deformed model into the standard commutative topological gauge theory. In section 4 we show that the action enjoys an invariance that reduces to ordinary diffeomorphism invariance in the commutative case. In section 5 we obtain solutions of the equations of motion and discuss their dependence on the noncommutativity parameter. Finally we present our conclusions.

\footnotetext{
${ }^{1}$ For other attempts to formulate gravity on noncommutative spaces based on Chern-Simons actions and its variants cf. 12$]$ 16].
} 


\section{Deformation of two-dimensional topological gauge theory}

It is well-known that the JT model [9] of dilaton gravity in two dimensions can be formulated as an $S U(1,1)$ topological gauge theory [10,11]. This is similar to the threedimensional case, where pure Einstein gravity can be written as a Chern-Simons theory [17. In what follows, we will use this gauge theory formulation to define 2- $d$ noncommutative gravity. To this end, we first note that the group $S U(1,1)$ is not closed with respect to the Moyal product, and thus we are forced to consider a gauge theory based on $U(1,1)$. The action of the $U(1,1)$ gauge theory on noncommutative $\mathbb{R}^{2}$, with coordinates $x^{\mu}$ satisfying $\left[x^{\mu}, x^{\nu}\right]=i \theta^{\mu \nu}$, reads ${ }^{2}$

$$
S=\beta \int \operatorname{Tr}(\Phi \star F)
$$

where $\beta$ is a dimensionless coupling constant. $\Phi=\Phi^{A} \tau_{A}, A=A_{\mu}^{A} \tau_{A} d x^{\mu}$ and $F=$ $\frac{1}{2} F_{\mu \nu}^{A} \tau_{A} d x^{\mu} \wedge d x^{\nu}$ take values in the Lie algebra $u(1,1)$ (the generators $\tau_{A}$ are given in the appendix). The star denotes the usual Moyal product, and

$$
\begin{aligned}
F & =D A=d A+A \wedge^{\star} A, \\
& \Downarrow \\
F_{\mu \nu} & =\partial_{\mu} A_{\nu}-\partial_{\nu} A_{\mu}+A_{\mu} \star A_{\nu}-A_{\nu} \star A_{\mu} .
\end{aligned}
$$

Note that the fields $\Phi^{A}, A_{\mu}^{A}$ and $F_{\mu \nu}^{A}$ are real, and that this reality is preserved under the infinitesimal gauge transformations

$$
\delta_{\lambda} A=d \lambda+[A, \lambda]=D \lambda, \quad \delta_{\lambda} \Phi=[\Phi, \lambda]
$$

where $\lambda=\lambda^{A} \tau_{A}$. The integrated form of (2.3) is given by

$$
A \rightarrow g_{\star}^{-1} \star A \star g+g_{\star}^{-1} \star d g, \quad \Phi \rightarrow g_{\star}^{-1} \star \Phi \star g,
$$

with $g=\exp _{\star} \lambda \in U(1,1)_{\star}$, i. e. , $g_{\star}^{-1}=\eta g^{\dagger} \eta$, where $\eta=\operatorname{diag}(-1,1)$ and $g \star g_{\star}^{-1}=1=$ $g_{\star}^{-1} \star g$.

The equations of motion following from (2.1) read

\footnotetext{
${ }^{2}$ Of course in two dimensions $\left[x^{\mu}, x^{\nu}\right]=i \theta^{\mu \nu}$ implies timelike noncommutativity, so one might think that problems like causality violation or loss of unitarity [18, 19] occur. However, as we will see below (cf. Eq. (2.5)), the deformed theory is still topological, so that there are no propagating degrees of freedom, and we thus expect the theory to be well-defined in spite of timelike noncommutativity.

${ }^{3}$ In what follows, all commutators and anticommutators are taken with respect to the Moyal product.
} 


$$
\begin{aligned}
F & =0, \\
D \Phi & =d \Phi+[A, \Phi]=0,
\end{aligned}
$$

so that the solutions are those of flat $U(1,1)$ connections in two dimensions. The equation (2.5) implies that locally one can write

$$
A_{\mu}=g_{\star}^{-1} \star \partial_{\mu} g
$$

with $g \in U(1,1)_{\star}$. Using

$$
\partial_{\mu} g=i \theta_{\mu \nu}\left[g, x^{\nu}\right]
$$

where $\theta_{\mu \nu} \theta^{\nu \lambda}=\delta_{\mu}^{\lambda}$, and defining the covariant coordinates

$$
X^{\mu}=x^{\mu}+i \theta^{\mu \nu} A_{\nu}
$$

one immediately obtains

$$
X^{\mu}=g_{\star}^{-1} \star x^{\mu} \star g .
$$

Note that the $X^{\mu}$ satisfy $\left[X^{\mu}, X^{\nu}\right]=i \theta^{\mu \nu}$. We still have to solve Eq. (2.6), which is equivalent to $\left[\Phi, X^{\mu}\right]=0$. Inserting (2.10) and the ansatz $\Phi=g^{-1} \star B \star g$, where $B \in u(1,1)$, one gets $\partial_{\mu} B=0$, so $B$ is constant. The solution of (2.6) is thus

$$
\Phi=g_{\star}^{-1} \star B \star g .
$$

In order to make contact with gravity, we decompose the $u(1,1)$ valued scalar and gauge fields according to

$$
\Phi^{A}=\left(\phi^{a}, \phi, \rho\right), \quad A_{\mu}^{A}=\left(e_{\mu}^{a} / l, \omega_{\mu}, b_{\mu}\right),
$$

where $a=0,1$ and $l$ is related to the negative cosmological constant by $\Lambda=-1 / l^{2}$. We note that in the noncommutative case one is forced to include the fields $\rho$ and $b_{\mu}$, which correspond to the trace part of $\mathrm{u}(1,1)$, in addition to the usual spin connection $\omega_{\mu}=\omega_{01 \mu}$, the zweibein $e_{\mu}^{a}$, and the scalars $\phi$ and $\phi^{a}$.

In what follows it will be convenient to define 


$$
\begin{aligned}
\Omega_{\mu b}^{a} & =\epsilon_{b}^{a} \omega_{\mu}+i \delta_{b}^{a} b_{\mu} \\
T_{\mu \nu}^{a} & =\partial_{\mu} e_{\nu}^{a}-\partial_{\nu} e_{\mu}^{a}+\frac{1}{2}\left[\Omega_{\mu b}^{a}, e_{\nu}^{b}\right]-\frac{1}{2}\left[\Omega_{\nu b}^{a}, e_{\mu}^{b}\right] \\
\phi_{a b} & =\phi \epsilon_{a b}-i \rho \eta_{a b}
\end{aligned}
$$

where

$$
\left[\Omega_{\mu b}^{a}, e_{\nu}^{b}\right] \equiv \Omega_{\mu b}^{a} \star e_{\nu}^{b}-e_{\nu}^{b} \star \Omega_{\mu b}^{a} .
$$

We will see below that $\Omega^{a}{ }_{b}$ can be interpreted as a noncommutative $s o(1,1) \oplus u(1)$ spin connection, with a trace part given by the abelian gauge field $b_{\mu}$, whereas $T^{a}$ is the noncommutative torsion.

Using the decomposition (2.12) and (2.13) - (2.15), the action (2.1) can be written as

$$
S=\frac{\beta}{4} \int d^{2} x \epsilon^{\mu \nu}\left[\phi_{a b} \star\left(\mathcal{R}_{\mu \nu}^{a b}-2 \Lambda e_{[\mu}^{a} \star e_{\nu]}^{b}\right)-2 \phi_{a} \star T_{\mu \nu}^{a}\right]
$$

where we defined the noncommutative curvature two-form

$$
\mathcal{R}_{\mu \nu}^{a b}=\partial_{\mu} \Omega_{\nu}^{a b}-\partial_{\nu} \Omega_{\mu}^{a b}+\frac{1}{2}\left[\Omega_{\mu c}^{a}, \Omega_{\nu}^{c b}\right]-\frac{1}{2}\left[\Omega_{\nu c}^{a}, \Omega_{\mu}^{c b}\right]
$$

from which we can see that indeed $\Omega^{a}{ }_{b}$ plays the role of an $s o(1,1) \oplus u(1)$ spin connection. The action (2.17) defines our model of noncommutative gravity in two dimensions.

As in the commutative case, the fields $\phi^{a}$ are Lagrange multipliers imposing the constraint $T_{\mu \nu}^{a}=0$, i. e., the vanishing of the noncommutative torsion.

The other equations of motion following from (2.17) read

$$
\begin{aligned}
\mathcal{R}_{b \mu \nu}^{a}-\frac{1}{2 l^{2}} \epsilon_{b}^{a} \epsilon_{c d}\left\{e_{\mu}^{c}, e_{\nu}^{d}\right\}+\frac{1}{2 l^{2}} \delta^{a}{ }_{b} \eta_{c d}\left[e_{\mu}^{c}, e_{\nu}^{d}\right] & =0, \\
\partial_{\nu} \phi_{a b}+\frac{1}{2}\left(\Omega_{\nu a}^{c} \star \phi_{c b}-\phi_{a c} \star \Omega_{\nu b}{ }^{c}\right)-\frac{1}{2} \epsilon_{a b} \epsilon_{c d}\left\{e_{\nu}^{c}, \phi^{d}\right\}-\frac{1}{2} \eta_{a b} \eta_{c d}\left[e_{\nu}^{c}, \phi^{d}\right] & =0, \\
\partial_{\nu} \phi^{a}+\frac{1}{2}\left(\Omega_{\nu b}^{a} \star \phi^{b}-\phi^{b} \star \Omega_{\nu b}{ }^{a}\right)+\frac{1}{2 l}\left(\phi_{b}{ }^{a} \star e_{\nu}^{b}-e_{\nu}^{b} \star \phi_{b}{ }_{b}\right) & =0 .
\end{aligned}
$$

Now one can construct a metric according to

$$
G_{\mu \nu}=e_{\mu}^{a} \star e_{\nu}^{b} \eta_{a b}=g_{\mu \nu}+i B_{\mu \nu}
$$

where 


$$
g_{\mu \nu}=\frac{1}{2} \eta_{a b}\left\{e_{\mu}^{a}, e_{\nu}^{b}\right\}
$$

is real and symmetric, and reduces to the usual expression for the metric in the commutative case, whereas

$$
B_{\mu \nu}=-\frac{i}{2} \eta_{a b}\left[e_{\mu}^{a}, e_{\nu}^{b}\right]
$$

is real and antisymmetric, and vanishes for $\theta^{\mu \nu}=0$.

We finally note that the action (2.1) can be rewritten as a matrix model for the covariant coordinates $X^{\mu}$ defined in (2.9). Setting $\theta=\theta^{01}$, the action reads

$$
S=-\frac{\beta}{2 \theta^{2}} \epsilon_{\mu \nu} \operatorname{Tr}_{\mathcal{H}}\left(\Phi \star\left(\left[X^{\mu}, X^{\nu}\right]-i \theta^{\mu \nu}\right)\right)
$$

Here $\Phi$ and $X^{\mu}$ are operators acting on a Hilbert space $\mathcal{H}=\mathcal{H}^{\prime} \otimes V$, where $\mathcal{H}^{\prime}$ is the infinite-dimensional subspace of the Hilbert space carrying the irreducible representation of $\left[x^{\mu}, x^{\nu}\right]=i \theta^{\mu \nu}$, whereas $V$ carries the fundamental representation of $u(1,1)$ [2].

From (2.23) we see that the scalar $\Phi$ is a Lagrange multiplier enforcing noncommutativity of the covariant coordinates,

$$
\left[X^{\mu}, X^{\nu}\right]=i \theta^{\mu \nu}
$$

and $\theta$ enters the coupling constant. It is interesting to note that, by generalizing the map from $u(2)$ to $u(1)$ gauge models constructed in [20] to a correspondence between $u(1,1)$ and $u(1)$, one can represent the $u(1,1)$ valued functions $\Phi$ and $X^{\mu}$ as scalar functions in a $u(1)$ theory?.

\section{The Seiberg-Witten map}

In [22] it was shown that the Seiberg-Witten formula maps the Chern-Simons (CS) action on noncommutative spaces into the standard commutative Chern-Simons action. As the commutative version of the topological gauge theory (2.1) can be obtained from the CS theory by dimensional reduction, one might ask whether a similar property holds for (2.1), i. e. , whether it is related to the standard commutative topological gauge theory by the Seiberg-Witten map. We will now show that this is indeed the case.

\footnotetext{
${ }^{4}$ Cf. also [21].

${ }^{5}$ Note however that due to the modified group metric in our case $(U(1,1)$ rather than $U(2))$ this mapping is less trivial than the one in [20], and would produce a nonstandard $U(1)$ action. We would like to thank A. Polychronakos for comments on this.
} 
A correspondence between commutative and noncommutative gauge field theories can be defined by the SW map [1]

$$
\begin{aligned}
\delta \Phi & =-\frac{i}{4} \delta \theta^{\alpha \beta}\left\{A_{\alpha}, \partial_{\beta} \Phi+D_{\beta} \Phi\right\}, \\
\delta F_{\mu \nu} & =\frac{i}{4} \delta \theta^{\alpha \beta}\left[2\left\{F_{\mu \alpha}, F_{\nu \beta}\right\}-\left\{A_{\alpha}, \partial_{\beta} F_{\mu \nu}+D_{\beta} F_{\mu \nu}\right\}\right],
\end{aligned}
$$

where the transformation formula for the adjoint scalar $\Phi$ can be found by dimensional reduction from three to two dimensions, setting $\theta^{2 \mu}=0, \mu=0,1$, and $\Phi=A_{3}$.

In order to study the variation of the action (2.1) under the SW map, we differentiate it with respect to $\theta^{\alpha \beta}$,

$$
\frac{\delta S}{\delta \theta^{\alpha \beta}}=-\frac{\beta}{2} \int d^{2} x \epsilon^{\mu \nu} \operatorname{Tr}\left(\frac{\delta F_{\mu \nu}}{\delta \theta^{\alpha \beta}} \star \Phi+F_{\mu \nu} \star \frac{\delta \Phi}{\delta \theta^{\alpha \beta}}\right) .
$$

Using (3.1), this simplifies after some algebra to

$$
\frac{\delta S}{\delta \theta^{\alpha \beta}}=\frac{\beta}{2} \int d^{2} x \operatorname{Tr}\left[-\frac{i}{4}\left\{F_{\alpha \beta}, \Phi\right\} \star F_{01}-\frac{i}{4} \Phi \star\left\{F_{0 \alpha}, F_{\beta 1}\right\}+\frac{i}{4} \Phi \star\left\{F_{0 \beta}, F_{\alpha 1}\right\}\right] .
$$

As we are in two dimensions, the only nonvanishing component of $\theta^{\mu \nu}$ is $\theta^{01}=\theta$. It is then easy to see that

$$
\frac{\delta S}{\delta \theta}=0
$$

and therefore the deformed action is mapped to the standard commutative one. We have to keep in mind, however, that the SW map is of perturbative nature in $\theta$, so the equivalence between the deformed and undeformed models holds perturbatively. Of course, the noncommutative theory (2.1) can also have solitonic solutions that become singular for $\theta \rightarrow 0$, and thus have no analogue in the commutative case. Furthermore, in deriving (3.4), we discarded boundary terms, so that in presence of a boundary the equivalence of the noncommutative and the commutative topological gauge theory fails. This is analogous to the case of Chern-Simons theory in three dimensions [22.

\section{Gauge symmetries and deformed diffeomorphisms}

In this section we want to find a candidate for diffeomorphisms in the noncommutative case 7 . Since in the commutative limit we want to obtain standard results, we start from

${ }^{6}$ For an alternative derivation of the SW equation cf. [23.

${ }^{7}$ For related work cf. [24. 
what is known in that case. The commutative version of our action (2.1) is invariant not only under the commutative version of the gauge transformations (2.3), but also under infinitesimal diffeomorphisms (Lie derivatives) along an arbitrary vector field $v=v^{\mu} \partial_{\mu}$,

$$
\begin{aligned}
\mathcal{L}_{v} A & =\left(d i_{v}+i_{v} d\right) A \\
\mathcal{L}_{v} \Phi & =i_{v} d \Phi,
\end{aligned}
$$

where $i_{v}$ is the inner product on differential forms.

Using the Leibnitz rule for the inner product $\left(\omega^{p}\right.$ and $\xi^{q}$ are respectively a $\mathrm{p}$ - and a q-form),

$$
i_{v}\left(\omega^{p} \wedge \xi^{q}\right)=\left(i_{v} \omega^{p}\right) \wedge \xi^{q}+(-)^{p} \omega^{p} \wedge\left(i_{v} \xi^{q}\right)
$$

it is easy to prove that

$$
\begin{aligned}
& \mathcal{L}_{v} A=\delta_{i_{v} A} A+i_{v} F, \\
& \mathcal{L}_{v} \Phi=\delta_{i_{v} A} \Phi+i_{v} D \Phi .
\end{aligned}
$$

From Eq. (4.3) and from the equations of motion (2.5-2.6) we see that, on-shell, the infinitesimal diffeomorphisms can be written as gauge transformations with parameters $\lambda=i_{v} A$. This fact can be used to relate translations and gauge transformations with $\lambda=\alpha^{a} \tau_{a}$. To this end we divide the connection into a part containing the zweibein and a part containing the spin connection and the center $U(1)$,

$$
A=l^{-1} e+\Theta, e=e_{\mu}^{a} \tau_{a} d x^{\mu}, \Theta=\omega_{\mu} \tau_{2} d x^{\mu}+b_{\mu} \tau_{3} d x^{\mu} .
$$

In this way, with an invertible $e_{\mu}^{a}$, we can write

$$
v^{\mu}:=l e_{a}^{\mu} \alpha^{a} \Leftrightarrow \alpha^{a}=l^{-1} i_{v} e^{a},
$$

thus obtaining

$$
\begin{aligned}
& \delta_{\alpha^{a} \tau_{a}} A \doteq \mathcal{L}_{v} A+\delta_{i_{v} \Theta} A \\
& \delta_{\alpha^{a} \tau_{a}} \Phi \doteq \mathcal{L}_{v} \Phi+\delta_{i_{v} \Theta} \Phi
\end{aligned}
$$

In the noncommutative case the situation is quite different. Whereas the action (2.1) is invariant under gauge transformations (2.3), the invariance under diffeomorphisms seems

\footnotetext{
${ }^{8}$ We use the symbol $\doteq$ for equations which are valid on-shell.
} 
to be completely destroyed. However we will show that the results obtained in the commutative case naturally suggest how to deform the diffeomorphism invariances in the noncommutative case.

Let us introduce the most natural generalization of the inner product and the Lie derivative in the noncommutative theory. If $\omega^{p}$ is a p-form we define

$$
\begin{aligned}
& \omega^{p}=\frac{1}{p !} \omega_{\mu_{1} \ldots \mu_{p}} d x^{\mu_{1}} \wedge \ldots \wedge d x^{\mu_{p}} \\
& i_{v}^{\star} \omega^{p}:=\frac{1}{2(p-1) !}\left[v^{\rho} \star \omega_{\rho \mu_{1} \ldots \mu_{p-1}}^{p}+\omega_{\rho \mu_{1} \ldots \mu_{p-1}}^{p} \star v^{\rho}\right] d x^{\mu_{1}} \wedge \ldots \wedge d x^{\mu_{p-1}}, \\
& \mathcal{L}_{v}^{\star} \omega^{p}:=\frac{1}{2 p !}\left\{\left[v^{\rho} \star \partial_{\rho} \omega_{\mu_{1} \ldots \mu_{p}}^{p}+\left(\partial_{\mu_{1}} v^{\rho}\right) \star \omega_{\rho \mu_{2} \ldots \mu_{p}}^{p}+\ldots+\left(\partial_{\mu_{p}} v^{\rho}\right) \star \omega_{\mu_{1} \ldots \mu_{p-1} \rho}^{p}\right]\right. \\
& +\left[\left(\partial_{\rho} \omega_{\mu_{1} \ldots \mu_{p}}^{p}\right) \star v^{\rho}+\omega_{\rho \mu_{2} \ldots \mu_{p}}^{p} \star \partial_{\mu_{1}} v^{\rho}+\ldots\right. \\
& \left.\left.+\omega_{\mu_{1} \ldots \mu_{p-1} \rho}^{p} \star \partial_{\mu_{p}} v^{\rho}\right]\right\} d x^{\mu_{1}} \wedge \ldots \wedge d x^{\mu_{p}} \\
& =\left(d i_{v}^{\star}+i_{v}^{\star} d\right) \omega^{p}
\end{aligned}
$$

where the exterior derivative $d$ is defined as in the commutative case and satisfies the same properties. It is important to note that the Leibnitz rule in Eq. (4.2) is not valid anymore. Using the above definitions we get

$$
\begin{aligned}
i_{v}^{\star}\left[A \wedge^{\star} A\right] & =\left(i_{v}^{\star} A\right) \star A-A \star\left(i_{v}^{\star} A\right)+\frac{1}{2}\left\{A_{\mu},\left[A, v^{\mu}\right]\right\}, \\
i_{v}^{\star}[\Phi \star A] & =\Phi \star\left(i_{v}^{\star} A\right)-\frac{1}{2}\left[\Phi, v^{\mu}\right] \star A_{\mu}, \\
i_{v}^{\star}[A \star \Phi] & =\left(i_{v}^{\star} A\right) \star \Phi+\frac{1}{2} A_{\mu} \star\left[\Phi, v^{\mu}\right] .
\end{aligned}
$$

Inspired by the commutative counterpart in (4.3) we define the deformed diffeomorphisms as

$$
\begin{aligned}
\Delta_{v}^{\star} & :=i_{v}^{\star} D+\delta_{i_{v}^{\star} A}, \\
& \Downarrow \\
\Delta_{v}^{\star} A & =i_{v}^{\star} F+\delta_{i_{v}^{\star} A} A, \\
\Delta_{v}^{\star} \Phi & =i_{v}^{\star} D \Phi+\delta_{i_{v}^{\star} A} \Phi .
\end{aligned}
$$

Now we want to prove that these transformations are symmetries of the action modulo boundary terms. First we note that, if $\xi$ is a 1-form and $\omega$ is a two form, a noncommutative integrated version of the Leibnitz rule (4.2) is valid:

$$
\int \xi \wedge^{\star} i_{v}^{\star} \omega=\int d^{2} x \epsilon^{\mu \nu} \xi_{\mu} \star\left(i_{v}^{\star} \omega\right)_{\nu}
$$




$$
\begin{aligned}
& =\frac{1}{2} \int d^{2} x \epsilon^{\mu \nu} \xi_{\nu} \star\left(v^{\rho} \star \omega_{\rho \nu}+\omega_{\rho \nu} \star v^{\rho}\right) \\
& =\frac{1}{2} \int d^{2} x \epsilon^{\mu \nu}\left(v^{\rho} \star \xi_{\mu}+\omega_{\mu} \star v^{\rho}\right) \star \omega_{\rho \nu} \\
& =\frac{1}{4} \int d^{2} x \epsilon^{\mu \nu}\left(v^{\rho} \star \xi_{\rho}+\omega_{\rho} \star v^{\rho}\right) \star \omega_{\mu \nu} \\
& =\int\left(i_{v}^{\star} \xi\right) \star \omega .
\end{aligned}
$$

Note that in order to obtain (4.10) the symmetric form of the definition of $i^{\star}$ and the fact that we are in two dimensions are crucial.

Since we know that the action is gauge invariant under (2.3), in order to prove its invariance under the deformed diffeomorphisms (4.9) it is sufficient to show that it remains unaltered by the transformations

$$
\begin{aligned}
& \delta_{v}^{\prime} A=i_{v}^{\star} F, \\
& \delta_{v}^{\prime} \Phi=i_{v}^{\star} D \Phi .
\end{aligned}
$$

Using Eq.(4.10), we obtain

$$
\begin{aligned}
\delta_{v}^{\prime} S & =\beta \int \operatorname{Tr}\left[i^{\star}(D \Phi) \star F+\Phi \star\left(d i_{v}^{\star} F+i_{v}^{\star} F \wedge^{\star} A+A \wedge^{\star} i_{v}^{\star} F\right)\right] \\
& =\beta \int \operatorname{Tr}\left(D \Phi \wedge^{\star} i_{v}^{\star} F-d \Phi \wedge^{\star} i_{v}^{\star} F+\Phi \star i_{v}^{\star} F \wedge^{\star} A+\Phi \star A \wedge^{\star} i_{v}^{\star} F\right) \\
& =\beta \int \operatorname{Tr}\left[D \Phi \wedge^{\star} i_{v}^{\star} F-(d \Phi+A \star \Phi-\Phi \star A) \wedge^{\star} i_{v}^{\star} F\right] \\
& =0
\end{aligned}
$$

Thus we have shown that the deformed diffeomorphisms (4.14) are indeed symmetries of the action.

We can also write the noncommutative version of Eq. (4.6) as

$$
\begin{aligned}
& \delta_{\alpha^{a} \tau_{a}} A \doteq \Delta_{v}^{\star} A+\delta_{i_{v}^{\star} \Theta} A \\
& \delta_{\alpha^{a} \tau_{a}} \Phi \doteq \Delta_{v}^{\star} \Phi+\delta_{i_{v}^{\star} \Theta} \Phi
\end{aligned}
$$

where now $v=v^{\mu} \partial_{\mu}$ is such that $\alpha^{a}=l^{-1} i_{v}^{\star} e^{a}$. Note that the vector $v$ can always be chosen to be real. This is shown in appendix $\mathrm{Q}$.

Using Eq.(4.8), it is straightforward to prove that the deformed diffeomorphisms (4.9) can be written as

$$
\Delta_{v}^{\star}=\mathcal{L}_{v}^{\star}+\frac{1}{2}\left\{A_{\mu},\left[,, v^{\mu}\right]\right\}
$$


The transformation properties of the fields (2.12) under the action of (4.14) are given in appendix $\mathrm{D}$. In particular, the symmetric part $g_{\mu \nu}$ and the antisymmetric part $B_{\mu \nu}$ of the metric (2.20) transform as

$$
\begin{aligned}
\Delta_{v}^{\star} g_{\mu \nu}= & \mathcal{L}_{v}^{\star} g_{\mu \nu}+\frac{1}{4} \eta_{a b}\left(\left[\left[e_{\mu}^{a}, v^{\rho}\right], \partial_{\rho} e_{\nu}^{b}\right]+\left[\left[e_{\nu}^{a}, v^{\rho}\right], \partial_{\rho} e_{\mu}^{b}\right]\right. \\
& \left.+\left[\left[e_{\mu}^{a}, \partial_{\nu} v^{\rho}\right], e_{\rho}^{b}\right]+\left[\left[e_{\nu}^{a}, \partial_{\mu} v^{\rho}\right], e_{\rho}^{b}\right]\right) \\
& +\frac{1}{8} \epsilon_{a b}\left(\left\{e_{\mu}^{a},\left[\omega_{\rho},\left[e_{\nu}^{b}, v^{\rho}\right]\right]\right\}+\left\{e_{\nu}^{a},\left[\omega_{\rho},\left[e_{\mu}^{b}, v^{\rho}\right]\right]\right\}\right. \\
& \left.-\left\{e_{\mu}^{a},\left[e_{\rho}^{b},\left[b_{\nu}, v^{\rho}\right]\right]\right\}-\left\{e_{\nu}^{a},\left[e_{\rho}^{b},\left[b_{\mu}, v^{\rho}\right]\right]\right\}\right), \\
\Delta_{v}^{\star} B_{\mu \nu}= & \mathcal{L}_{v}^{\star} B_{\mu \nu}-\frac{i}{4} \eta_{a b}\left(\left\{\left[e_{\mu}^{a}, v^{\rho}\right], \partial_{\rho} e_{\nu}^{b}\right\}-\left\{\left[e_{\nu}^{a}, v^{\rho}\right], \partial_{\rho} e_{\mu}^{b}\right\}\right. \\
& \left.+\left\{\left[e_{\mu}^{a}, \partial_{\nu} v^{\rho}\right], e_{\rho}^{b}\right\}-\left\{\left[e_{\nu}^{a}, \partial_{\mu} v^{\rho}\right], e_{\rho}^{b}\right\}\right) \\
& -\frac{i}{8} \epsilon_{a b}\left(\left[e_{\mu}^{a},\left[\omega_{\rho},\left[e_{\nu}^{b}, v^{\rho}\right]\right]\right]-\left[e_{\nu}^{a},\left[\omega_{\rho},\left[e_{\mu}^{b}, v^{\rho}\right]\right]\right]\right. \\
& \left.-\left[e_{\mu}^{a},\left[e_{\rho}^{b},\left[b_{\nu}, v^{\rho}\right]\right]\right]+\left[e_{\nu}^{a},\left[e_{\rho}^{b},\left[b_{\mu}, v^{\rho}\right]\right]\right]\right) .
\end{aligned}
$$

One may note that these transformations reduce to ordinary diffeomorphisms if $\theta^{\mu \nu}=0$.

\section{Some solutions}

\subsection{Fuzzy AdS}

The undeformed gravitational action admits the $\mathrm{AdS}_{2}$ solution

$$
d s^{2}=-\frac{r^{2}}{l^{2}} d t^{2}+\frac{l^{2}}{r^{2}} d r^{2}
$$

This leads to the connection

$$
A_{t}=\frac{i r}{2 l^{2}}\left(\begin{array}{cc}
1 & 1 \\
-1 & -1
\end{array}\right), \quad A_{r}=\frac{1}{2 r}\left(\begin{array}{ll}
0 & 1 \\
1 & 0
\end{array}\right)
$$

which can be written as $A=g^{-1} d g$ with

$$
g=\frac{1}{2} \sqrt{\frac{r}{l}}\left(\begin{array}{cc}
1+\frac{i t}{2 l} & \frac{i t}{2 l} \\
-\frac{i t}{2 l} & 1-\frac{i t}{2 l}
\end{array}\right)\left(\begin{array}{cc}
1+\frac{l}{r} & 1-\frac{l}{r} \\
1-\frac{l}{r} & 1+\frac{l}{r}
\end{array}\right) .
$$

At this point, we observe that $g$ is the product of two matrices $f, h \in S U(1,1)$, each one depending on a single variable only. This implies that $f, h$ are elements of $U(1,1)_{\star}$. Consequently we have $\tilde{g}:=f \star h \in U(1,1)_{\star}$ and we can use $\tilde{g}$ to obtain a noncommutative 
solution $\tilde{A}_{\mu}=\tilde{g}_{\star}^{-1} \partial_{\mu} \tilde{g}$.

With these matrices, we easily obtain

$$
\tilde{A}_{\mu}=A_{\mu},
$$

so that (5.1) is a solution of the noncommutative gravity. This is the fuzzy $\mathrm{AdS}_{2}$. Fuzzy $\mathrm{AdS}_{2}$ was obtained in [25] by an analytic continuation of the fuzzy sphere [27]. Let us briefly recall the construction. We denote the Cartesian coordinates of $\mathrm{AdS}_{2}$ by $X^{-1}, X^{0}, X^{1}$. The algebra of fuzzy $\mathrm{AdS}_{2}$ is [25]

$$
\begin{aligned}
{\left[X^{-1}, X^{0}\right] } & =-i \theta l^{-1} X^{1} \\
{\left[X^{0}, X^{1}\right] } & =i \theta l^{-1} X^{-1} \\
{\left[X^{1}, X^{-1}\right] } & =i \theta l^{-1} X^{0}
\end{aligned}
$$

where $\theta$ is the noncommutativity parameter, and $l$ the curvature radius of $\mathrm{AdS}_{2}$, so

$$
\eta_{i j} X^{i} X^{j}=-\left(X^{-1}\right)^{2}-\left(X^{0}\right)^{2}+\left(X^{1}\right)^{2}=-l^{2},
$$

with $\left(\eta_{i j}\right)=\operatorname{diag}(-1,-1,1)$. The isometry group $S U(1,1)$ of $\mathrm{AdS}_{2}$ preserves the algebra (5.5), and thus $S U(1,1)$ is also a symmetry of fuzzy $\mathrm{AdS}_{2}$.

In the commutative case $\theta \rightarrow 0$, the $X^{i}$ are commuting coordinates and one can parametrize $\mathrm{AdS}_{2}$ by

$$
r=X^{-1}+X^{1}, \quad t=\frac{l}{r} X^{0} .
$$

This leads to the induced metric (5.1).

In the noncommutative case, (5.7) suggests the definition

$$
r=X^{-1}+X^{1}, \quad t=\frac{l}{2}\left(r^{-1} X^{0}+X^{0} r^{-1}\right),
$$

where we have introduced symmetrized products for $r^{-1}$ and $X^{0}$ so that $t$ is a Hermitian operator [25]. From (5.5) it follows that the commutation relation for $t$ and $r$ is given by

$$
[t, r]=i \theta .
$$

Further evidence for (5.9) was given in [26] by considering closed strings in $\mathrm{AdS}_{2}$. Besides, we note that (5.9) is preserved by diffeomorphisms generated by the three Killing vectors of the metric (5.1).

\footnotetext{
${ }^{9}$ Cf. also [26].
} 
Finally we observe that, writing the $\mathrm{AdS}_{2}$ metric in a conformally flat form by introducing $x=l^{2} / r$,

$$
d s^{2}=\frac{l^{2}}{x^{2}}\left(-d t^{2}+d x^{2}\right),
$$

the new coordinates obey the commutation relation

$$
[x, t]=i \theta l^{-2} x^{2},
$$

which is that of a quantum plane structure.

\subsection{Deformed solutions}

Besides the hermitian metric defined in equation (2.20), there is another fundamental bitensor which can be constructed in a natural way and corresponds to a nonantisymmetric volume 2-form:

$$
E_{\mu \nu}=\epsilon_{a b} e_{\mu}^{a} \star e_{\nu}^{b}=H_{\mu \nu}+i M_{\mu \nu}
$$

where

$$
H_{\mu \nu}=\frac{1}{2} \epsilon_{a b}\left\{e_{\mu}^{a}, e_{\nu}^{b}\right\}
$$

is real and antisymmetric and reduce to the usual volume form in the commutative case, whereas

$$
M_{\mu \nu}=-\frac{i}{2} \epsilon_{a b}\left[e_{\mu}^{a}, e_{\nu}^{b}\right]
$$

is real and symmetric and vanishes for $\theta^{\mu \nu}=0$.

We observe that, while in the commutative case the tensors $G_{\mu \nu}$ and $E_{\mu \nu}$ are invariant under the gauge transformations corresponding to the boost and the $U(1)$ gauge symmetry, in the noncommutative case this invariance property is no longer valid. Using Eq.(B.2), it is easy to show that under an infinitesimal boost these fields transform as

$$
\delta_{\xi \tau_{2}} G_{\mu \nu}=-\frac{1}{2}\left[E_{\mu \nu}, \xi\right], \quad \delta_{\xi \tau_{2}} E_{\mu \nu}=-\frac{1}{2}\left[G_{\mu \nu}, \xi\right] .
$$

The fields $g_{\mu \nu}, B_{\mu \nu}, H_{\mu \nu}$ and $M_{\mu \nu}$ transform according to 


$$
\begin{aligned}
\delta_{\xi \tau_{2}} g_{\mu \nu} & =-\frac{i}{2}\left[M_{\mu \nu}, \xi\right], & \delta_{\xi \tau_{2}} B_{\mu \nu} & =\frac{i}{2}\left[H_{\mu \nu}, \xi\right], \\
\delta_{\xi \tau_{2}} H_{\mu \nu} & =-\frac{i}{2}\left[B_{\mu \nu}, \xi\right], & \delta_{\xi \tau_{2}} M_{\mu \nu} & =\frac{i}{2}\left[g_{\mu \nu}, \xi\right] .
\end{aligned}
$$

For an infinitesimal $U(1)$ gauge transformation, we obtain from Eq. (B.3) the transformation rules

$$
\delta_{\chi \tau_{3}} G_{\mu \nu}=\frac{i}{2}\left[G_{\mu \nu}, \chi\right], \quad \delta_{\chi \tau_{3}} E_{\mu \nu}=\frac{i}{2}\left[E_{\mu \nu}, \chi\right]
$$

or equivalently

$$
\begin{aligned}
\delta_{\chi \tau_{3}} g_{\mu \nu} & =\frac{i}{2}\left[g_{\mu \nu}, \chi\right], & \delta_{\chi \tau_{3}} B_{\mu \nu} & =\frac{i}{2}\left[B_{\mu \nu}, \chi\right], \\
\delta_{\chi \tau_{3}} H_{\mu \nu} & =\frac{i}{2}\left[H_{\mu \nu}, \chi\right], & \delta_{\chi \tau_{3}} M_{\mu \nu} & =\frac{i}{2}\left[M_{\mu \nu}, \chi\right] .
\end{aligned}
$$

We see that in the noncommutative case all the fields become charged under the $U(1)$ gauge symmetry.

These results can be summarized and generalized to finite gauge transformations assembling $G_{\mu \nu}$ and $E_{\mu \nu}$ together in a doublet,

$$
S_{\mu \nu}:=\left(\begin{array}{c}
G_{\mu \nu} \\
E_{\mu \nu}
\end{array}\right)=\left(\begin{array}{c}
g_{\mu \nu} \\
H_{\mu \nu}
\end{array}\right)+i\left(\begin{array}{c}
B_{\mu \nu} \\
M_{\mu \nu}
\end{array}\right),
$$

which transforms under a finite boost or $U(1)$ gauge transformation as

$$
S_{\mu \nu}^{T} \longmapsto\left[U^{-1} \star S_{\mu \nu}\right]^{T} \star U
$$

In order to deform the classical $\mathrm{AdS}_{2}$ solution (5.1), we can iterate Eq. (5.16) twice, obtaining, up to second order in $\theta=\theta^{01}$

$$
\begin{aligned}
g_{t t} & =-\frac{r^{2}}{l^{2}}+\theta^{2} \frac{r}{2 l^{2}}\left[\partial_{r} \partial_{t} \xi \partial_{t} \xi-\partial_{t}^{2} \xi \partial_{r} \xi+r^{-1} \partial_{t} \xi \partial_{t} \xi\right]+\mathcal{O}\left(\theta^{3}\right), \\
g_{r r} & =\frac{l^{2}}{r^{2}}+\theta^{2} \frac{l^{2}}{2 r^{3}}\left[\partial_{r} \partial_{t} \xi \partial_{t} \xi-\partial_{t}^{2} \xi \partial_{r} \xi-3 r^{-1} \partial_{t} \xi \partial_{t} \xi\right]+\mathcal{O}\left(\theta^{3}\right), \\
M_{t t} & =-\theta \frac{r}{l^{2}} \partial_{t} \xi+\mathcal{O}\left(\theta^{3}\right), \\
M_{r r} & =-\theta \frac{l^{2}}{r^{3}} \partial_{t} \xi+\mathcal{O}\left(\theta^{3}\right),
\end{aligned}
$$


where $\xi(t, r)$ is an arbitrary function, which should of course be restricted appropriately if one wants the metric to approach $\mathrm{AdS}_{2}$ asymtotically, i. e. , for $r \rightarrow \infty$. Note that the antisymmetric volume form $H_{\mu \nu}$, as well as $B_{\mu \nu}$, continue to be zero.

We further observe that corrections to the metric become very large when $r \rightarrow 0$, so that perturbative calculations in the noncommutativity parameter $\theta$ are not reliable near the horizon.

\section{Conclusions}

In this paper, we presented a model of noncommutative gravity in two dimensions based on a deformation of an $S U(1,1)$ topological gauge theory. The substitution of ordinary products by the Moyal product makes it necessary to enlarge the gauge group to $U(1,1)$. This amounts to introducing an additional abelian gauge field as well as an additional scalar. Those fields corresponding to the trace part are coupled to the gravitational fields in the noncommutative case, and decouple for $\theta^{\mu \nu}=0$. We also showed that the deformed action admits an equivalent formulation in terms of a matrix model, whose coupling constant contains the noncommutativity parameter.

The metric that we defined contains a symmetric part, which reduces to the ordinary metric once the noncommutativity parameter is set to zero, and an antisymmetric part that vanishes for $\theta^{\mu \nu}=0$.

Furthermore, some solutions of the noncommutative model, like fuzzy $\mathrm{AdS}_{2}$, were obtained, and symmetries of the deformed action were studied. In particular, it was found that the action is invariant under a class of transformations that reduce to ordinary diffeomorphisms in the commutative case.

We saw that the Seiberg-Witten formula maps the topological gauge theory on noncommutative spaces to the standard commutative one. This behaviour is known from three-dimensional Chern-Simons theory [22], and might be related to the topological nature of the model. One has to keep in mind, however, that the Seiberg-Witten map is of perturbative nature in $\theta$, and that the deformed action can admit solitonic solutions that become singular when the noncommutativity parameter tends to zero. Therefore, we do not expect the deformed model to be entirely equivalent to the undeformed one. Further development of our work would be to address issues like the quantization of the theory, or the classification of solutions of the matrix model (2.23). We hope to report on this in the near future.

\section{Acknowledgements}

This work was partially supported by INFN, MURST and by the European Commission RTN program HPRN-CT-2000-00131, in which the S. C. , D. K. , L. M. and D. Z. are associated to the University of Torino. 


\section{A Conventions}

An element $M$ of the Lie algebra $u(1,1)$ satisfies

$$
M_{b}^{a}=-\eta_{b c} \bar{M}_{d}^{c} \eta^{d a}
$$

where a bar denotes complex conjugation, and $\left(\eta_{a b}\right)=\operatorname{diag}(-1,1)$. We choose as $u(1,1)$ generators

$$
\begin{array}{rlrl}
\tau_{0} & =\frac{1}{2}\left(\begin{array}{cc}
i & 0 \\
0 & -i
\end{array}\right), & \tau_{1}=\frac{1}{2}\left(\begin{array}{ll}
0 & 1 \\
1 & 0
\end{array}\right), \\
\tau_{2}=\frac{1}{2}\left(\begin{array}{cc}
0 & -i \\
i & 0
\end{array}\right), & \tau_{3}=\frac{1}{2}\left(\begin{array}{cc}
i & 0 \\
0 & i
\end{array}\right) .
\end{array}
$$

They are normalized according to

$$
\operatorname{Tr}\left(\tau_{A} \tau_{B}\right)=\frac{1}{2} \eta_{A B}
$$

where $\left(\eta_{A B}\right)=\operatorname{diag}(-1,1,1,-1)$ is the inner product on the Lie algebra. The generators (A.2) satisfy the relation (A.1). Further, if $i, j, k$ assume the values 0,1 and 2 , then the following relations hold:

$$
\begin{aligned}
{\left[\tau_{i}, \tau_{j}\right] } & =-\epsilon_{i j k} \tau^{k} \\
{\left[\tau_{i}, \tau_{3}\right] } & =0 \\
\tau_{i} \tau_{j} & =-\frac{1}{2} \epsilon_{i j k} \tau^{k}-\frac{i}{2} \eta_{i j} \tau_{3} \\
\operatorname{Tr}\left(\tau_{i} \tau_{j} \tau_{k}\right) & =-\frac{1}{4} \epsilon_{i j k} \\
\operatorname{Tr}\left(\tau_{i} \tau_{j} \tau_{3}\right) & =\frac{i}{4} \eta_{i j}
\end{aligned}
$$

where $\left(\eta_{i j}\right)=\operatorname{diag}(-1,1,1)$ and $\epsilon_{012}=1$.

We furthermore defined $\epsilon_{01}=1$, and antisymmetrize with unit weight, e. g.

$$
e_{[\mu}^{a} \star e_{\nu]}^{b} \equiv \frac{1}{2}\left(e_{\mu}^{a} \star e_{\nu}^{b}-e_{\nu}^{a} \star e_{\mu}^{b}\right)
$$




\section{B Gauge transformations of the gravitational fields}

In this appendix we write the explicit form of the different gauge transformation for the gravitational fields defined in Eq.(2.12). If we divide gauge transformations defined in Eq. (2.3) in translations $\left(\lambda=\alpha^{a} \tau_{a}\right)$, boost $\left(\lambda=\xi \tau_{2}\right)$ and $U(1)$ gauge symmetry $\left(\lambda=\chi \tau_{3}\right)$, their action on the fields turns out to be the following:

- Translations

$$
\begin{aligned}
\delta_{\alpha} e^{a} & =l d \alpha^{a}+\frac{l}{2} \epsilon_{b}^{a}\left\{\omega, \alpha^{b}\right\}+\frac{i l}{2}\left[b, \alpha^{a}\right], \\
\delta_{\alpha} \phi^{a} & =\frac{1}{2} \epsilon_{b}^{a}\left\{\phi, \alpha^{b}\right\}+\frac{i}{2}\left[\rho, \alpha^{a}\right], \\
\delta_{\alpha} \omega & =-\frac{1}{2 l} \epsilon_{a b}\left\{e^{a}, \alpha^{b}\right\}, \quad \delta_{\alpha} \phi=-\frac{1}{2} \epsilon_{a b}\left\{\phi^{a}, \alpha^{b}\right\}, \\
\delta_{\alpha} b & =-\frac{i}{2 l} \eta_{a b}\left[e^{a}, \alpha^{b}\right], \quad \delta_{\alpha} \rho=-\frac{i}{2 l} \eta_{a b}\left[\phi^{a}, \alpha^{b}\right] .
\end{aligned}
$$

- Boost

$$
\begin{aligned}
\delta_{\xi} e^{a} & =-\frac{1}{2} \epsilon_{b}^{a}\left\{e^{b}, \xi\right\}, & \delta_{\xi} \phi^{a} & =-\frac{1}{2} \epsilon^{a}{ }_{b}\left\{\phi^{b}, \xi\right\}, \\
\delta_{\xi} \omega & =d \xi+\frac{i}{2}[b, \xi], & \delta_{\xi} \phi & =\frac{i}{2}[\rho, \xi], \\
\delta_{\xi} b & =-\frac{i}{2}[\omega, \xi], & \delta_{\xi} \rho & =-\frac{i}{2}[\phi, \xi] .
\end{aligned}
$$

- $\mathrm{U}(1)$ gauge symmetry

$$
\begin{aligned}
\delta_{\chi} e^{a} & =\frac{i}{2}\left[e^{a}, \chi\right], & \delta_{\chi} \phi^{a} & =\frac{i}{2}\left[\phi^{a}, \chi\right], \\
\delta_{\chi} \omega & =\frac{i}{2}[\omega, \chi], & \delta_{\chi} \phi & =\frac{i}{2}[\phi, \chi], \\
\delta_{\chi} b=d \chi & +\frac{i}{2}[b, \chi], & \delta_{\chi} \rho & =\frac{i}{2}[\rho, \chi] .
\end{aligned}
$$

\section{Reality of the vector field $v$}

We still have to prove that the vector field $v$ generating the transformations (4.9) can always be chosen to be real. Indeed assuming the zweibein $e_{\mu}^{a}$ to be invertible as an ordinary matrix, we look for a solution $v$ of $l \alpha^{a}=i_{v}^{\star} e^{a}=v^{\mu} \star_{S} e_{\mu}^{a}$, with $\star_{S}:=\cos \left(\frac{1}{2} \overleftarrow{\partial} \times \partial \vec{\partial}\right)$ written as a series in $\theta^{\mu \nu}$, 


$$
v=\sum_{n=0}^{\infty} \stackrel{(n)}{v},
$$

where $\stackrel{(n)}{v}$ is of order $n$ in $\theta^{\mu \nu}$.

Now we write

$$
\begin{aligned}
l \alpha^{a} & =v^{\mu} \star_{S} e_{\mu}^{a} \\
& =\sum_{n, m=0}^{\infty} \frac{(-)^{m}}{(2 m) !} \stackrel{(n)}{v}^{(2 m}\left(\frac{1}{2} \overleftarrow{\partial} \times \vec{\partial}\right)^{2 m} e_{\mu}^{a} \\
& =\sum_{n, m=0}^{\infty} \frac{(-)^{m}}{(2 m) !}\left[{ }^{(2 n)} \mu\left(\frac{1}{2} \overleftarrow{\partial} \times \vec{\partial}\right)^{2 m} e_{\mu}^{a}+\stackrel{(2 n+1)}{v} \mu\left(\frac{1}{2} \overleftarrow{\partial} \times \vec{\partial}\right)^{2 m} e_{\mu}^{a}\right] \\
& =\sum_{k=0}^{\infty} \sum_{m=0}^{k} \frac{(-)^{m}}{(2 m) !}\left[\begin{array}{c}
(2 k-2 m) \\
v
\end{array}\left(\frac{1}{2} \overleftarrow{\partial} \times \vec{\partial}\right)^{2 m} e_{\mu}^{a}+\stackrel{(2 k-2 m+1)}{a}\left(\frac{1}{2} \overleftarrow{\partial} \times \vec{\partial}\right)^{2 m} e_{\mu}^{a}\right]
\end{aligned}
$$

We find that the zero order condition admits a unique solution

$$
\stackrel{(0)}{v}_{a}^{\mu} e_{\mu}^{a}=\alpha^{a}
$$

whereas the first order condition

$$
\stackrel{(1)}{v}^{\mu} e_{\mu}^{a}=0
$$

implies that $\stackrel{(1)}{v}^{\mu} \equiv 0$. By induction, knowing $\stackrel{(n)}{v} \mu$ for every $n \leq 2 k$, the $\stackrel{(2 k+2)}{v}^{\mu}$ and $\stackrel{(2 k+3)}{v} \mu$ are uniquely determined in terms respectively of the $v^{(2 n)} \mu$ and $\stackrel{(2 n+1)}{v} \mu$ of lower order. So, if $\stackrel{(1)}{v}^{\mu} \equiv 0$, then ${ }^{(2 n+1)}{ }^{\mu} \equiv 0$ for every $n$ and knowing ${ }_{v}^{(0)} \mu$ one can construct iteratively the complete solution of the condition (C.2).

\section{Transformation properties of the fields}

Under the action of the transformations (4.14), the fields (2.12) transform as

$$
\begin{aligned}
\Delta_{v}^{\star} e_{\mu}^{a}= & \mathcal{L}_{v}^{\star} e_{\mu}^{a}+\frac{1}{4} \epsilon_{b}^{a}\left(\left[\omega_{\nu},\left[e_{\mu}^{b}, v^{\nu}\right]\right]-\left[e_{\nu}^{b},\left[b_{\mu}, v^{\nu}\right]\right]\right) \\
& +\frac{i}{4}\left(\left\{e_{\nu}^{a},\left[b_{\mu}, v^{\nu}\right]\right\}+\left\{b_{\nu},\left[e_{\mu}^{a}, v^{\nu}\right]\right\}\right)
\end{aligned}
$$




$$
\begin{aligned}
\Delta_{v}^{\star} \omega_{\mu}= & \mathcal{L}_{v}^{\star} \omega_{\mu}-\frac{1}{4 l^{2}} \epsilon_{a b}\left[e_{\nu}^{a},\left[e_{\mu}^{b}, v^{\nu}\right]\right] \\
& +\frac{i}{4}\left(\left\{\omega_{\nu},\left[b_{\mu}, v^{\nu}\right]\right\}+\left\{b_{\nu},\left[\omega_{\mu}, v^{\nu}\right]\right\}\right) \\
\Delta_{v}^{\star} b_{\mu}= & \mathcal{L}_{v}^{\star} b_{\mu}-\frac{i}{4 l^{2}}\left(\eta_{a b}\left\{e_{\nu}^{a},\left[e_{\mu}^{b}, v^{\nu}\right]\right\}+\left\{\omega_{\nu},\left[\omega_{\mu}, v^{\nu}\right]\right\}\right) \\
& +\frac{i}{4}\left\{b_{\nu},\left[b_{\mu}, v^{\nu}\right]\right\} \\
\Delta_{v}^{\star} \phi^{a}= & \mathcal{L}_{v}^{\star} \phi^{a}+\frac{1}{4} \epsilon^{a}{ }_{b}\left(\left[\omega_{\nu},\left[\phi^{b}, v^{\nu}\right]\right]-\frac{1}{l}\left[e_{\nu}^{b},\left[\phi, v^{\nu}\right]\right]\right) \\
& +\frac{i}{4}\left(\frac{1}{l}\left\{e_{\nu}^{a},\left[\rho, v^{\nu}\right]\right\}+\left\{b_{\nu},\left[\phi^{a}, v^{\nu}\right]\right\}\right) \\
\Delta_{v}^{\star} \phi= & \mathcal{L}_{v}^{\star} \omega_{\mu}-\frac{1}{4 l} \epsilon_{a b}\left[e_{\nu}^{a},\left[\phi^{b}, v^{\nu}\right]\right] \\
& +\frac{i}{4}\left(\left\{\omega_{\nu},\left[\rho, v^{\nu}\right]\right\}+\left\{b_{\nu},\left[\phi, v^{\nu}\right]\right\}\right) \\
& +\frac{i}{4}\left\{b_{\nu},\left[\rho, v^{\nu}\right]\right\} . \\
\Delta_{v}^{\star} \rho= & \mathcal{L}_{v}^{\star} \rho-\frac{i}{4}\left(\frac{1}{l} \eta_{a b}\left\{e_{\nu}^{a},\left[\phi^{b}, v^{\nu}\right]\right\}+\left\{\omega_{\nu},\left[\phi, v^{\nu}\right]\right\}\right) \\
&
\end{aligned}
$$

18 


\section{References}

[1] N. Seiberg and E. Witten, "String theory and noncommutative geometry," JHEP 9909 (1999) 032 arXiv:hep-th/9908142.

[2] M. R. Douglas and N. A. Nekrasov, "Noncommutative field theory," Rev. Mod. Phys. 73 (2002) 977 arXiv:hep-th/0106048.

[3] A. H. Chamseddine, "Deforming Einstein's gravity," Phys. Lett. B 504 (2001) 33 arXiv:hep-th/0009153.

[4] A. H. Chamseddine, "Complexified gravity in noncommutative spaces," Commun. Math. Phys. 218 (2001) 283 arXiv:hep-th/0005222.

[5] A. H. Chamseddine, "Complex gravity and noncommutative geometry," Int. J. Mod. Phys. A 16 (2001) 759 arXiv:hep-th/0010268.

[6] H. Nishino and S. Rajpoot, "Teleparallel complex gravity as foundation for noncommutative gravity," arXiv:hep-th/0107216.

[7] T. Damour, S. Deser and J. G. McCarthy, "Nonsymmetric gravity theories: Inconsistencies and a cure," Phys. Rev. D 47 (1993) 1541 arXiv:gr-qc/9207003.

[8] J. W. Moffat, "Nonsymmetric Gravitational Theory," J. Math. Phys. 36 (1995) 3722 [Erratum-ibid. 36 (1995) 7128]; "Noncommutative quantum gravity," Phys. Lett. B 491 (2000) 345 arXiv:hep-th/0007181; "Perturbative noncommutative quantum gravity," Phys. Lett. B 493 (2000) 142 [arXiv:hep-th/0008089].

[9] C. Teitelboim, "Gravitation And Hamiltonian Structure In Two Space-Time Dimensions," Phys. Lett. B 126 (1983) 41, and "The Hamiltonian structure of twodimensional space-time and its relation with the conformal anomaly," in Quantum Theory of Gravity, S. Christensen, ed. (Adam Hilger, Bristol, 1984);

R. Jackiw, "Liouville field theory: a two-dimensional model for gravity?," in Quantum Theory of Gravity, S. Christensen, ed. (Adam Hilger, Bristol, 1984), and "Lower Dimensional Gravity," Nucl. Phys. B 252 (1985) 343.

[10] K. Isler and C. A. Trugenberger, "A Gauge Theory Of Two-Dimensional Quantum Gravity," Phys. Rev. Lett. 63 (1989) 834.

[11] A. H. Chamseddine and D. Wyler, "Topological Gravity In (1+1)-Dimensions," Nucl. Phys. B 340 (1990) 595.

[12] M. Bañados, O. Chandia, N. Grandi, F. A. Schaposnik and G. A. Silva, "Threedimensional noncommutative gravity," Phys. Rev. D 64 (2001) 084012 arXiv:hepth/0104264.

[13] V. P. Nair, "Gravitational fields on a noncommutative space," arXiv:hep-th/0112114. 
[14] S. Cacciatori, D. Klemm, L. Martucci and D. Zanon, "Noncommutative Einstein-AdS gravity in three dimensions," arXiv:hep-th/0201103.

[15] K. Shiraishi, "Noncommutative gravity in three dimensions coupled to point-like sources," arXiv:hep-th/0202099.

[16] A. H. Chamseddine, "Invariant actions for noncommutative gravity," arXiv:hepth/0202137.

[17] A. Achucarro and P. K. Townsend, "A Chern-Simons Action For Three-Dimensional Anti-De Sitter Supergravity Theories," Phys. Lett. B 180 (1986) 89;

E. Witten, "(2+1)-Dimensional Gravity As An Exactly Soluble System," Nucl. Phys. B 311 (1988) 46.

[18] N. Seiberg, L. Susskind and N. Toumbas, "Space/time non-commutativity and causality," JHEP 0006 (2000) 044 arXiv:hep-th/0005015.

[19] J. Gomis and T. Mehen, "Space-time noncommutative field theories and unitarity," Nucl. Phys. B 591 (2000) 265 arXiv:hep-th/0005129.

[20] V. P. Nair and A. P. Polychronakos, "On level quantization for the noncommutative Chern-Simons theory," Phys. Rev. Lett. 87 (2001) 030403 arXiv:hep-th/0102181.

[21] E. Kiritsis and C. Sochichiu, "Duality in non-commutative gauge theories as a nonperturbative Seiberg-Witten map," arXiv:hep-th/0202065.

[22] N. Grandi and G. A. Silva, "Chern-Simons action in noncommutative space," Phys. Lett. B 507 (2001) 345 arXiv:hep-th/0010113.

[23] A. A. Bichl, J. M. Grimstrup, H. Grosse, E. Kraus, L. Popp, M. Schweda and R. Wulkenhaar, "Noncommutative Lorentz symmetry and the origin of the SeibergWitten map," arXiv:hep-th/0108045;

J. M. Grimstrup, H. Grosse, E. Kraus, L. Popp, M. Schweda and R. Wulkenhaar, "Noncommutative spin-1/2 representations," arXiv:hep-th/0110231.

[24] R. Jackiw and S. Y. Pi, "Covariant coordinate transformations on noncommutative space," arXiv:hep-th/0111122.

[25] P. M. Ho and M. Li, "Fuzzy spheres in AdS/CFT correspondence and holography from noncommutativity," Nucl. Phys. B 596 (2001) 259 arXiv:hep-th/0004072.

[26] P. M. Ho and M. Li, "Large N expansion from fuzzy AdS 2 ," Nucl. Phys. B 590 (2000) 198 arXiv:hep-th/0005268].

[27] J. Madore, "The Fuzzy sphere," Class. Quant. Grav. 9 (1992) 69. 